\title{
INVERSE PROBLEMS FOR REGULARIZATION MATRICES
}

\author{
SILVIA NOSCHESE* AND LOTHAR REICHEL ${ }^{\dagger}$ \\ Dedicated to Claude Brezinski and Sebastiano Seatzu \\ on the Occasion of Their 70th Birthdays.
}

\begin{abstract}
Discrete ill-posed problems are difficult to solve, because their solution is very sensitive to errors in the data and to round-off errors introduced during the solution process. Tikhonov regularization replaces the given discrete ill-posed problem by a nearby penalized least-squares problem whose solution is less sensitive to perturbations. The penalization term is defined by a regularization matrix, whose choice may affect the quality of the computed solution significantly. We describe several inverse matrix problems whose solution yields regularization matrices adapted to the desired solution. Numerical examples illustrate the performance of the regularization matrices determined.
\end{abstract}

1. Introduction. We consider the computation of an approximate solution of minimization problems of the form

$$
\min _{x \in \mathbb{C}^{n}}\|A x-b\|_{2},
$$

where $\|\cdot\|_{2}$ denotes the Euclidean vector norm and $A \in \mathbb{C}^{m \times n}$ is a matrix with many singular values of different orders of magnitude close to the origin. Minimization problems (1.1) with a matrix of this kind are commonly referred to as discrete illposed problems. They arise, for example, from the discretization of linear ill-posed problems, such as Fredholm integral equations of the first kind. In discrete ill-posed problems that arise in applications in science and engineering, the vector $b \in \mathbb{C}^{m}$ in (1.1) represents error-contaminated data. We will for notational simplicity assume that $m \geq n$.

Let $e \in \mathbb{C}^{m}$ denote the (unknown) error in $b$, and let $\hat{b} \in \mathbb{C}^{m}$ be the error-free vector associated with $b$, i.e.,

$$
b=\hat{b}+e .
$$

The unavailable linear system of equations with error-free right-hand side,

$$
A x=\hat{b}
$$

is assumed to be consistent. Let $A^{\dagger}$ denote the Moore-Penrose pseudoinverse of $A$. We are interested in determining an approximation of the solution $\hat{x}=A^{\dagger} \hat{b}$ of minimal Euclidean norm of the unavailable linear system (1.3) by computing an approximate solution of the available least-squares problem (1.1). Note that the solution of (1.1),

$$
\breve{x}=A^{\dagger} b=A^{\dagger}(\hat{b}+e)=\hat{x}+A^{\dagger} e,
$$

typically is dominated by the propagated error $A^{\dagger} e$ and therefore is not useful.

\footnotetext{
* Dipartimento di Matematica "Guido Castelnuovo", SAPIENZA Università di Roma, P.le A. Moro, 2, I-00185 Roma, Italy. E-mail: noschese@mat.uniroma1.it. Research supported by a grant from SAPIENZA Università di Roma.

$\dagger$ Department of Mathematical Sciences, Kent State University, Kent, OH 44242, USA. E-mail: reichel@math.kent.edu. Research supported in part by NSF grant DMS-1115385.
} 
Tikhonov regularization seeks to determine an accurate approximation of $\hat{x}$ by replacing the minimization problem (1.1) by a penalized least-squares problem of the form

$$
\min _{x \in \mathbb{C}^{n}}\left\{\|A x-b\|_{2}^{2}+\mu\|L x\|_{2}^{2}\right\}
$$

where the matrix $L \in \mathbb{C}^{h \times n}, h \leq n$, is referred to as the regularization matrix and the scalar $\mu>0$ is the regularization parameter. The matrix $L$ is chosen so that the null spaces of $A$ and $L$ intersect trivially. Then the minimization problem (1.5) has the unique solution

$$
x_{L, \mu}=\left(A^{T} A+\mu L^{T} L\right)^{-1} A^{T} b
$$

for any $\mu>0$. The purpose of the regularization term $\mu\|L x\|_{2}^{2}$ in (1.5) is to damp the term $A^{\dagger} e$ in (1.4) and thereby obtain an accurate approximation of $\hat{x}$. Common regularization matrices $L$ are the identity matrix $I$ and finite difference matrices, such as

$$
L=\frac{1}{2}\left[\begin{array}{rrrrc}
1 & -1 & & & 0 \\
& 1 & -1 & & \\
& & \ddots & \ddots & \\
0 & & & 1 & -1
\end{array}\right] \in \mathbb{R}^{(n-1) \times n}
$$

and

$$
L=\frac{1}{4}\left[\begin{array}{rrrrrr}
-1 & 2 & -1 & & & 0 \\
& -1 & 2 & -1 & & \\
& & \ddots & \ddots & \ddots & \\
0 & & & -1 & 2 & -1
\end{array}\right] \in \mathbb{R}^{(n-2) \times n} .
$$

The quality of the approximation (1.6) of $\hat{x}$ depends both on the choice of the regularization matrix $L$ and on the size of $\mu>0$. It is the purpose of the matrix $L$ to reduce the propagated error $A^{\dagger} e$ without damping important features of the desired solution $\hat{x}$. For instance, the regularization matrix (1.8) is well suited for discrete ill-posed problems (1.1) that are discretizations of Fredholm integral equations of the first kind in one space-dimension and whose desired solution $\hat{x}$ is the discretization at equidistant nodes of a real-valued function in one space-variable with a large linear component, because vectors that represent discretizations at equidistant nodes of linear functions in one space-variable are in the null space of $L$. They therefore are not damped by $L$ in (1.5). Several approaches to construct regularization matrices are discussed in $[2,5,8,13,20]$.

This paper describes a novel approach to determine regularization matrices of simple form that do not dampen an available approximation of $\hat{x}$ much. Computed examples of Section 3 illustrate that Tikhonov regularization (1.5) with these regularization matrices can give more accurate approximations of $\hat{x}$ than standard regularization matrices, such as $L=I,(1.7)$, or (1.8). Specifically, we first solve (1.5) with $L=I$ for the approximation $x_{I, \mu}$ of $\hat{x}$. Then an inverse matrix problem is solved for $L$, where we require $L$ to be a square or trapezoidal tridiagonal matrix or a trapezoidal banded Toeplitz matrix such that $\left\|L x_{I, \mu}\right\|$ is small. Finally, we solve (1.5) with the computed regularization matrix. This yields an improved approximation $x_{L, \mu}$ of $\hat{x}$. 
The process may be repeated, i.e., a new regularization matrix that does not dampen $x_{L, \mu}$ can be constructed and then used to compute a better approximation of $\hat{x}$.

The regularization parameter $\mu$ in (1.5) can be determined in a variety of ways, such as by the discrepancy principle, extrapolation, generalized cross validation, or the L-curve; see, e.g., $[3,4,7,8,12]$ for discussions of these methods.

It is convenient to denote Toeplitz matrices in $\mathbb{C}^{n \times n}$ with bandwidth $2 k+1$ by

$$
T_{(k)}=(n ; k ; \sigma, \delta, \tau)=\left[\begin{array}{ccccccc}
\delta & \tau_{1} & \ldots & \tau_{k} & & 0 \\
\sigma_{1} & & & & & \\
\vdots & & & & \ddots & \\
\sigma_{k} & & & \ddots & & \\
& & \ddots & & & \\
& \ddots & & & & & \tau_{k} \\
& & & & & \vdots \\
0 & & & & & & \tau_{k} \\
0 & & & \sigma_{1} & \delta
\end{array}\right] .
$$

Tridiagonal matrices in $\mathbb{C}^{n \times n}$ are denoted by

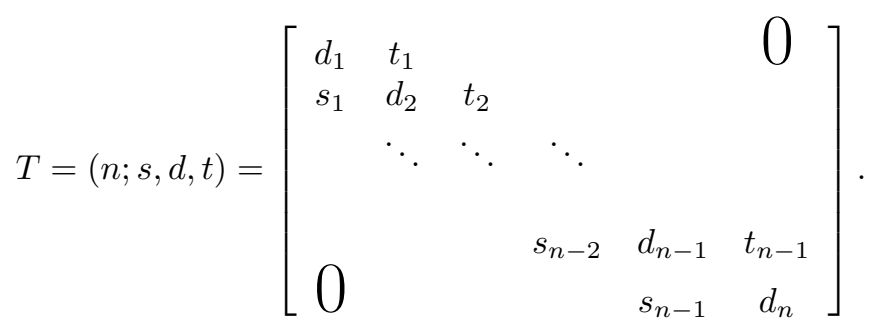

The performance of the regularization matrices of this paper can be understood in terms of certain of their $\varepsilon$-pseudoeigenvectors. For a given $\varepsilon>0$, the $\varepsilon$ pseudospectrum of the matrix $A \in \mathbb{C}^{n \times n}$ is the set

$$
\Lambda_{\varepsilon}(A)=\left\{z: \exists u \in \mathbb{C}^{n},\|u\|_{2}=1 \text {, such that }\|(z I-A) u\|_{2} \leq \varepsilon\right\},
$$

see, e.g., [22]. The vectors $u$ are referred to as $\varepsilon$-pseudoeigenvectors. The importance of these vectors is commented on at the end of Section 2 .

We remark that the eigenvalues of nonsymmetric irreducible tridiagonal matrices can be very sensitive to perturbations of the matrix. Also, the eigenvalues of large banded nonnormal Toeplitz matrices can be poorly conditioned; the condition number of the eigenvalues of such a matrix grows exponentially with the dimension $n$, except when the boundary of the spectrum of the associated Toeplitz operator is a curve with no interior. This curve is related to the $\varepsilon$-pseudospectrum of the matrix as $\varepsilon \rightarrow 0$ and $n \rightarrow \infty$; see [19, Theorem 3.2]. Spectral properties of normal and close to normal $(2 k+1)$-banded Toeplitz matrices of order $n$, with $k \leq\lfloor n / 2\rfloor$, are described in [16]. Normal and close to normal irreducible tridiagonal matrices have been analyzed in [14].

This paper is organized as follows. Section 2 describes several inverse matrix problems for regularization matrices. Computed examples with these regularization matrices are presented in Section 3. Concluding remarks can be found in Section 4. 
We finally note that choosing the regularization matrix adaptively, depending on the solution is not new. For instance, Tikhonov regularization is commonly used with a nonlinear regularization operator in image restoration; see, e.g., [6, 23]. A simplification of this approach is discussed in [18]. Recently, Huckle and Sedlacek [10, 11] described constructions of regularization matrices that depend on the magnitude of the components of the computed approximate solution [10] or on the matrix $A$. The approach of the present paper to determine a sequence of Toeplitz matrices is believed to be new.

2. Inverse matrix problems. This section describes several inverse problems for matrices with a banded Toeplitz or tridiagonal structure. The matrices are constructed so that a given vector, $x$, is an $\varepsilon$-pseudoeigenvector for a fairly small value of $\varepsilon>0$. The first problem determines a trapezoidal Toeplitz matrix by minimizing the norm of the matrix-vector product with a given vector.

Inverse Problem 1 Given a vector $x \in \mathbb{C}^{n}$, determine an upper trapezoidal banded Toeplitz matrix $B \in \mathbb{C}^{(n-2 k) \times n}$ with first row $\left[\sigma_{k}, \ldots, \sigma_{1}, 1, \tau_{1}, \ldots, \tau_{k}, 0, \ldots, 0\right]$ such that $B$ solves

$$
\min _{\sigma, \tau}\|B x\|_{2} .
$$

Let $x=\left[\xi_{1}, \xi_{2}, \ldots, \xi_{n}\right]^{T}$. Then the minimization problem (2.1) can be expressed as

$$
\min _{\sigma, \tau}\left\|\left[\begin{array}{cccccc}
\xi_{1} & \ldots & \xi_{k} & \xi_{k+2} & \ldots & \xi_{2 k+1} \\
\xi_{2} & \ldots & \xi_{k+1} & \xi_{k+3} & \ldots & \xi_{2 k+2} \\
\cdot & \cdot & \cdot & \cdot & \cdot & \cdot \\
\cdot & \cdot & \cdot & \cdot & \cdot & \cdot \\
\cdot & \cdot & \cdot & \cdot & \cdot & \cdot \\
\xi_{n-2 k} & \ldots & \xi_{n-k-1} & \xi_{n-k+1} & \ldots & \xi_{n}
\end{array}\right]\left[\begin{array}{c}
\sigma_{k} \\
\cdot \\
\sigma_{1} \\
\tau_{1} \\
\cdot \\
\tau_{k}
\end{array}\right]+\left[\begin{array}{c}
\xi_{k+1} \\
\xi_{k+2} \\
\cdot \\
\cdot \\
\cdot \\
\xi_{n-k}
\end{array}\right]\right\|_{2}
$$

This least-squares problem has a unique solution when the matrix in (2.2) has linearly independent columns. The following result holds.

Proposition 2.1. Two columns of the matrix in (2.2) are linearly dependent if and only if the components of $x$ for some $\alpha \in \mathbb{C}$ satisfy one of the following conditions:

1. $\exists s, t \in\{1, \ldots, k\}, s \neq t$, so that $\xi_{h+s-1}=\alpha \xi_{h+t-1}, h=1: n-2 k$;

2. $\exists s, t \in\{1, \ldots, k\}, s \neq t$, so that $\xi_{h+k+s}=\alpha \xi_{h+k+t}, h=1: n-2 k$;

3. $\exists s, t \in\{1, \ldots, k\}$ so that $\xi_{h+s-1}=\alpha \xi_{h+k+t}, h=1: n-2 k$.

Proof. If condition 1 [condition 2] holds, then two of the first [the last] $k$ columns are linearly dependent, whereas if condition 3 holds, then one of the first $k$ columns and one of the last $k$ columns are linearly dependent. Otherwise, all columns are linearly independent by twos.

The situation when $k=1$ has previously been discussed in [15]. In such a case $B$ is an upper trapezoidal tridiagonal Toeplitz matrix and, according to Proposition 2.1 , the two columns of the matrix in (2.2) are linearly dependent if and only if the components of $x$ satisfy

$$
\xi_{h}=\alpha \xi_{h+2}, \quad h=1: n-2,
$$

for some $\alpha \in \mathbb{C}$. Hence, in the tridiagonal case one has necessary and sufficient conditions for the unicity of the solution of the least-squares problem in (2.2). On the 
other hand, when $k>1$ it is difficult to provide transparent necessary conditions for having a unique solution of the minimization problem (2.1), Proposition 2.1 providing only non-unicity sufficient conditions.

When columns are linearly dependent we determine the unique solution of minimal Euclidean norm of the least-squares problem.

At the end of this section, we will refer to the square Toeplitz matrix with bandwidth $2 k+1$ obtained by prepending and appending suitable rows to $B$ as $T_{(k)}$; cf. (1.9).

The remainder of this section discusses inverse problems for tridiagonal matrices. The first two problems determine symmetric or skew-symmetric trapezoidal matrices.

Inverse Problem 2 Given a vector $x \in \mathbb{C}^{n}$, determine an upper trapezoidal tridiagonal matrix $S \in \mathbb{C}^{(n-2) \times n}$ with main diagonal of ones, and lower and upper diagonals $\left[s_{1}, \ldots, s_{n-1}\right]$ such that $S$ solves

$$
\min _{s}\|S x\|_{2} .
$$

We refer to $S$ as a symmetric trapezoidal tridiagonal matrix.

Let $x=\left[\xi_{1}, \xi_{2}, \ldots, \xi_{n}\right]^{T}$. Then the minimization problem (2.3) can be expressed as

$$
\min _{s}\left\|\left[\begin{array}{cccccc}
\xi_{1} & \xi_{3} & & & & \\
& \xi_{2} & \xi_{4} & & & \\
& & \cdot & \cdot & & \\
& & & \cdot & \cdot & \\
& & & & \cdot & \\
& & & & \xi_{n-2} & \xi_{n}
\end{array}\right]\left[\begin{array}{c}
s_{1} \\
s_{2} \\
\cdot \\
\cdot \\
\cdot \\
s_{n-1}
\end{array}\right]+\left[\begin{array}{c}
\xi_{2} \\
\xi_{3} \\
\cdot \\
\cdot \\
\cdot \\
\xi_{n-1}
\end{array}\right]\right\|_{2}
$$

The following result gives necessary and sufficient conditions for the existence of a unique solution of the minimization problem (2.3).

Proposition 2.2. The least square problem (2.4) has a unique solution if and only if all components of $x$ are nonvanishing except two nonconsecutive ones.

Proof. The matrix in (2.4) has $n-1$ columns and $n-2$ rows. Two columns are linearly dependent if and only if there exists a vanishing component $\xi_{j}$, with $j=2: n-1$. Additionally, the first [the last] column is vanishing if and only if $\xi_{1}=0\left[\xi_{n}=0\right]$. Finally, four columns from the second to the last but one are linearly dependent by twos if and only if two nonconsecutive components $\xi_{j}$, with $j=2: n-1$ are vanishing. Notice that any vanishing couple $\left(\xi_{j}, \xi_{j+1}\right), j=1: n-1$, only yields one vanishing column.

We denote the square symmetric tridiagonal matrix obtained by prepending and appending suitable rows to $S$ by $T=(n ; s, 1, s)$; cf. (1.10).

Inverse Problem 3 Given a vector $x \in \mathbb{C}^{n}$, determine an upper trapezoidal tridiagonal matrix $S \in \mathbb{C}^{(n-2) \times n}$ with lower diagonal $\left[s_{1}, \ldots, s_{n-1}\right]$, main diagonal of ones, and upper diagonal $\left[-s_{1}, \ldots,-s_{n-1}\right]$ such that $S$ solves

$$
\min _{s}\|S x\|_{2} .
$$

We refer to $S$ as a skew-symmetric trapezoidal tridiagonal matrix.

Let $x=\left[\xi_{1}, \xi_{2}, \ldots, \xi_{n}\right]^{T}$. Then the minimization problem (2.5) can be expressed as 


$$
\min _{s}\left\|\left[\begin{array}{cccccc}
\xi_{1} & -\xi_{3} & & & & \\
& \xi_{2} & -\xi_{4} & & & \\
& & \cdot & \cdot & & \\
& & & \cdot & & \\
& & & & \xi_{n-2} & -\xi_{n}
\end{array}\right]\left[\begin{array}{c}
s_{1} \\
s_{2} \\
\cdot \\
\cdot \\
\cdot \\
s_{n-1}
\end{array}\right]+\left[\begin{array}{c}
\xi_{2} \\
\xi_{3} \\
\cdot \\
\cdot \\
\cdot \\
\xi_{n-1}
\end{array}\right]\right\|_{2}
$$

The following result is strictly analogous to the one relevant to Inverse Problem 2 .

Proposition 2.3. The least square problem (2.6) has a unique solution if and only if all components of $x$ are nonvanishing except two nonconsecutive ones.

We denote the square skew-symmetric tridiagonal matrix obtained by prepending and appending suitable rows to $S$ by $T=(n ; s, 1,-s)$.

Inverse Problem 4 Given a vector $x \in \mathbb{C}^{n}$, determine a tridiagonal matrix $T=(n ; s, 1, t) \in \mathbb{C}^{n \times n}$ with lower diagonal $\left[s_{1}, \ldots, s_{n-1}\right]$, main diagonal of ones, and upper diagonal $\left[t_{1}, \ldots, t_{n-1}\right]$ such that $T$ solves

$$
\min _{s, t}\|T x\|_{2} \text {. }
$$

Let $x=\left[\xi_{1}, \xi_{2}, \ldots, \xi_{n}\right]^{T}$. Then the minimization problem (2.7) can be expressed as

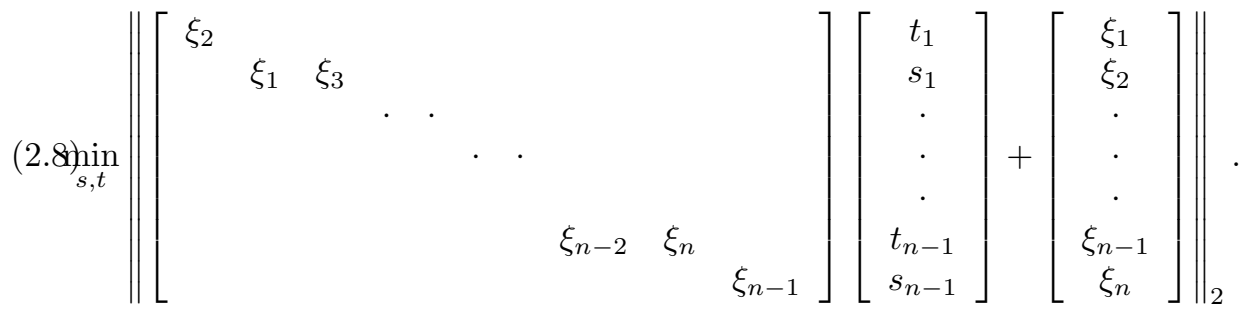

The following result give necessary and sufficient conditions for a unique solution to $(2.7)$.

Proposition 2.4. The least square problem (2.8) has a unique solution if and only if none of the couples $\left(\xi_{j}, \xi_{j+2}\right), j=0: n-1$ is vanishing, having assumed $\xi_{0}=\xi_{n+1}=0$.

Proof. The matrix in (2.8) has $2 n-2$ columns and $n$ rows. It has a vanishing row if and only if the components of $x$ satisfy one of the following conditions:

1. $\xi_{2}=0$

2. $\xi_{n-1}=0$;

3. $\exists h=1: n-2$ so that $\left(\xi_{h}, \xi_{h+2}\right)=(0,0)$.

Otherwise the matrix has full rank.

It follows from (1.11) that the $\varepsilon$-pseudoeigenvectors of $T_{(k)}$ [of $\left.T\right]$ associated with $z=0$ form a subset of

$$
\left\{u:\left\|T_{(k)} u\right\|_{2} \leq \varepsilon,\|u\|_{2}=1\right\}\left[\left\{u:\|T u\|_{2} \leq \varepsilon,\|u\|_{2}=1\right\}\right] .
$$

If zero is in the $\varepsilon$-pseudospectrum of $T_{(k)}$ [of $T$ ] for a small value of $\varepsilon>0$, then the corresponding $\varepsilon$-pseudoeigenvectors will be essentially undampened in the Tikhonov minimization problem (1.5). 
3. Computed examples. It is the purpose of this section to illustrate that the solutions of the inverse problems of Section 2 with $x$ an available approximate solution of (1.1), such as $x=x_{I, \mu}$, can be suitable regularization matrices for (1.5). The rationale for using the regularization matrix $L$ equal to one of the solution matrices in Section 2 is that we do not want the regularization matrix to damp important features of the desired solution $\hat{x}$ when solving (1.5). Ideally, we would like to solve one of the inverse problems of Section 2 with $x=\hat{x}$; however, since $\hat{x}$ is not known, we let $x$ be the best available approximation of $\hat{x}$. The examples below present application of this approach in an iterative fashion.

In the first three examples, we choose the regularization parameter that yields the smallest relative error $\left\|x_{L, \mu}-\hat{x}\right\|_{2} /\|\hat{x}\|_{2}$. This gives insight into how well the regularization matrices discussed may perform when a good method for determining the regularization parameter is used. The last example illustrates the performance of the method when the regularization parameter is determined with the aid of the discrepancy principle. We solve (1.5) for a general matrix $L$ by using the generalized singular value decomposition (GSVD) of the matrix pair $\{A, L\}$. It is then easy to evaluate $x_{L, \mu}$ for many values of $\mu$. When $L=I$, the GSVD can be replaced by the (standard) singular value decomposition (SVD) of $A$; see, e.g., Hansen [8] for details on the applications of the GSVD or SVD to the solution of (1.5). All computations were carried out in MATLAB with about 15 significant decimal digits.

Example 3.1. Consider the Fredholm integral equation of the first kind

$$
\int_{-6}^{6} \kappa(s, t) x(t) d t=g(s), \quad-6 \leq s \leq 6,
$$

where

$$
\phi(y)= \begin{cases}1+\cos \frac{\pi y}{3}, & |y|<3 \\ 0, & |y| \geq 3\end{cases}
$$

and the kernel $\kappa$ and the right-hand side $g$ are given by

$$
\begin{aligned}
\kappa(s, t) & =\phi(s-t), \\
g(s) & =(6-|s|)\left(1+\frac{1}{2} \cos \frac{\pi s}{3}\right)+\frac{9}{2 \pi} \sin \frac{\pi|s|}{3} .
\end{aligned}
$$

This equation is discussed by Phillips in [17]. We discretize the integral equation by a Galerkin method with orthonormal box functions as test and trial functions using the MATLAB function phillips from Regularization Tools [9]. The function yields $A \in \mathbb{R}^{200 \times 200}$ and a scaled approximation $\hat{x} \in \mathbb{R}^{200}$ of the solution $x(t)=\phi(t)$ of (3.1). The error-free right-hand side vector in (1.3) is computed as $\hat{b}=A \hat{x}$. The entries of the error $e$ in $b$ are normally distributed with zero mean, and they are scaled to correspond to $1 \%$ error; cf. (1.2).

We first compute the approximate solution $x_{I, \mu}$ of (1.1) by solving (1.5) with $L=I$ using the optimal value of the parameter $\mu>0$. This yields $\left\|x_{I, \mu}-\hat{x}\right\|_{2}=$ $1.031 \cdot 10^{-2}\|\hat{x}\|_{2}$.

Next we determine a tridiagonal regularization matrix $T \in \mathbb{R}^{200 \times 200}$ by solving Inverse Problem 4 with $x=x_{I, \mu}$. The regularization matrix $L=T$ so obtained is used in (1.5) to compute a new approximate solution, $x_{1}$, of (1.1). The vector $x_{1}$ is a better approximation of $\hat{x}$ than $x_{I, \mu}$; we have $\left\|x_{1}-\hat{x}\right\|_{2}=8.896 \cdot 10^{-3}\|\hat{x}\|_{2}$. We now can solve (2.7) with $x=x_{1}$ to determine a new tridiagonal regularization matrix 
$L=T$. Using the latter regularization matrix in (1.5) yields an improved approximate solution, $x_{2}$, of $\hat{x}$ with $\left\|x_{2}-\hat{x}\right\|_{2}=8.864 \cdot 10^{-3}\|\hat{x}\|_{2}$. Similarly, we compute $x_{3}$ with error $\left\|x_{3}-\hat{x}\right\|_{2}=8.850 \cdot 10^{-3}\|\hat{x}\|_{2}$ and $x_{4}$ with error $\left\|x_{4}-\hat{x}\right\|_{2}=8.847 \cdot 10^{-3}\|\hat{x}\|_{2}$.

The regularization matrix obtained by solving (2.7) generally is of better quality, the better the vector $x$ in (2.7) approximates $\hat{x}$. For instance, $x=\hat{x}$ gives a regularization matrix $L=T$ such that the error in the subsequently computed Tikhonov solution $x_{L, \mu}$ is $\left\|x_{L, \mu}-\hat{x}\right\|_{2}=6.326 \cdot 10^{-5}\|\hat{x}\|_{2}$.

Commonly used regularization matrices $L$ in (1.5) include the rectangular Toeplitz matrices (1.7) and (1.8). When using $L$ is defined by (1.7) with $n=200$, we obtain the approximate solution $x^{\prime}$ with error $\left\|x^{\prime}-\hat{x}\right\|_{2}=9.811 \cdot 10^{-3}\|\hat{x}\|_{2}$. Similarly, solving (1.5) with $L$ given by (1.8) yields the approximate solution $x^{\prime \prime}$ with $\left\|x^{\prime \prime}-\hat{x}\right\|_{2}=$ $1.021 \cdot 10^{-2}\|\hat{x}\|_{2}$. Thus, not only $x_{4}$, but also $x_{1}, x_{2}$ and $x_{3}$ are better approximations of $\hat{x}$ than $x^{\prime}$ and $x^{\prime \prime}$.

We may alternatively determine a tridiagonal regularization matrix $T \in \mathbb{R}^{198 \times 200}$ by solving Inverse Problem 2 with $x=x_{I, \mu}$. When the symmetric tridiagonal regularization matrix $L=T$ so obtained is used in (1.5) to compute a new approximate solution, $x_{1}$, we obtain $\left\|x_{1}-\hat{x}\right\|_{2}=9.982 \cdot 10^{-3}\|\hat{x}\|_{2}$. Solving (2.3) with $x=x_{1}$ determines a new symmetric tridiagonal regularization matrix $L=T$, which we use to compute the approximate solution $x_{2}$. The latter has the error $\left\|x_{2}-\hat{x}\right\|_{2}=9.766 \cdot 10^{-3}\|\hat{x}\|_{2}$. Similarly, we compute $x_{3}$, which has the error $\left\|x_{3}-\hat{x}\right\|_{2}=9.762 \cdot 10^{-3}\|\hat{x}\|_{2}$. Thus, $x_{3}$ is a better approximation of $\hat{x}$ than $x^{\prime}$ and $x^{\prime \prime}$. Thus, both Inverse Problems 2 and 4 gives good regularization matrices, with the latter inverse problem determining the ones that give the best approximations of $\hat{x}$ in this example.

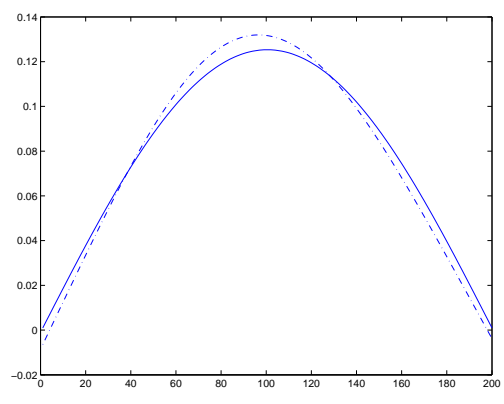

(a)

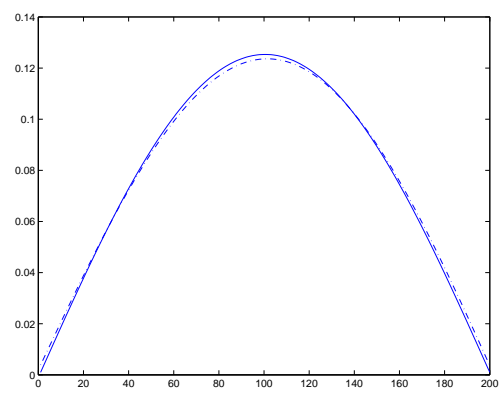

(b)

FIG. 3.1. Example 3.2. Solution $\hat{x}$ to the error-free problem (1.3) (solid curves) and computed approximations (dash-dotted curves); the approximate solutions are $x^{\prime \prime}$ in (a) and $x_{L, \mu}$ in (b).

Example 3.2. We consider the Fredholm integral equation of the first kind discussed by Baart [1]. Its kernel $\kappa$ and right-hand side $g$ are given by

$$
\kappa(s, t)=\exp (s \cos t), \quad g(s)=2 \frac{\sinh s}{s} .
$$

The interval of integration is $0 \leq t \leq \pi$ and $s \in\left[0, \frac{\pi}{2}\right]$. We discretize the integral equation using the MATLAB function baart from [9] to determine the matrix $A \in$ $\mathbb{R}^{200 \times 200}$ and a scaled approximation $\hat{x} \in \mathbb{R}^{200}$ of the solution $x(t)=\sin (t)$. We let $\hat{b}=A \hat{x}$. The error-contaminated vector $b$ is generated as in Example 3.1; it has $1 \%$ error. 
The approximate solution $x_{I, \mu}$ has the error $\left\|x_{I, \mu}-\hat{x}\right\|_{2}=1.764 \cdot 10^{-1}\|\hat{x}\|_{2}$. The rectangular tridiagonal Toeplitz regularization matrix $L=T_{(1)} \in \mathbb{R}^{198 \times 200}$ which solves Inverse Problem 1 with $k=1$ and $x=x_{I, \mu}$ is used in (1.5) to compute a new approximate solution, $x_{L, \mu}$, with error $\left\|x_{L, \mu}-\hat{x}\right\|_{2}=1.804 \cdot 10^{-2}\|\hat{x}\|_{2}$.

The rectangular tridiagonal Toeplitz matrix (1.8) with $n=200$ is known to work very well for the present problem; however, the error in the computed solution $x^{\prime \prime}$ obtained with the regularization matrix (1.8) is larger than for $x_{L, \mu}$. We have $\| x^{\prime \prime}-$ $\hat{x}\left\|_{2}=5.991 \cdot 10^{-2}\right\| \hat{x} \|_{2}$. Figures 3.1(a) and 3.1(b) display $x^{\prime \prime}$ and $x_{L, \mu}$, respectively.

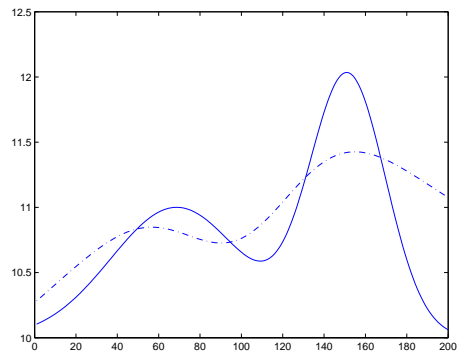

(a)

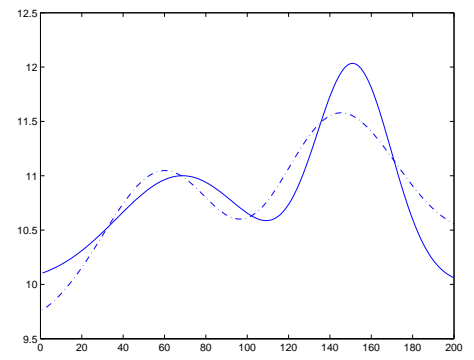

(b)

FIG. 3.2. Example 3.3. Solution $\hat{x}$ to the error-free problem (1.3) (solid curves) and computed approximations (dash-dotted curves); the approximate solutions are $x^{\prime \prime}$ in (a) and $x_{L, \mu}$ in (b).

Example 3.3. Consider the discretization of a Fredholm integral equation of the first kind with the kernel $\kappa$ and solution $x$ given by

$$
\begin{aligned}
\kappa(s, t) & =(\cos s+\cos t)^{2}\left(\frac{\sin u}{u}\right)^{2}, \quad u=\pi(\sin s+\sin t), \\
x(t) & =2 \exp \left(-6(t-0.8)^{2}\right)+\exp \left(-2(t+0.5)^{2}\right)+10 .
\end{aligned}
$$

The interval of integration is $t \in[-\pi / 2, \pi / 2]$; the variable $s$ lives in the same interval. A closely related integral equation is discussed by Shaw [21].

Discretization is carried out by a simple collocation method using the MATLAB code shaw from [9]. This yields the matrix $A \in \mathbb{R}^{200 \times 200}$ and discrete solution $\hat{x} \in$ $\mathbb{R}^{200}$. We define the error-free vector $\hat{b}=A \hat{x}$. The associated contaminated vector $b$ has $1 \%$ error and is defined as in Example 3.1.

Tikhonov regularization with $L=I$ gives a poor approximation of $\hat{x}$. Therefore, we use the regularization matrix (1.8) to determine the approximation $x^{\prime \prime}$ of $\hat{x}$. We obtain $\left\|x^{\prime \prime}-\hat{x}\right\|_{2}=3.622 \cdot 10^{-2}\|\hat{x}\|_{2}$.

Next we determine a tridiagonal regularization matrix $T \in \mathbb{R}^{200 \times 200}$ by solving Inverse Problem 4 with $x=x^{\prime \prime}$. The regularization matrix $L=T$ so obtained is used to compute a new approximate solution, $x_{L, \mu}$. This vector is a better approximation of $\hat{x}$ than $x^{\prime \prime}$; we have $\left\|x_{L, \mu}-\hat{x}\right\|_{2}=2.332 \cdot 10^{-2}\|\hat{x}\|_{2}$. Figures $3.2(\mathrm{a})$ and $3.2(\mathrm{~b})$ show $x^{\prime \prime}$ and $x_{L, \mu}$, respectively. We note also that $x=\hat{x}$ gives a regularization matrix $L=T$ such that the error in the subsequently computed Tikhonov solution $x_{L, \mu}$ is $\left\|x_{L, \mu}-\hat{x}\right\|_{2}=8.032 \cdot 10^{-6}\|\hat{x}\|_{2}$.

Example 3.4. Let the matrix $A$ be the same as in Example 3.2 and let the minimal-norm solution, $\hat{x}$, of the error-free problem (1.3) be obtained by adding 10 to every component of the corresponding solution of Example 3.2. Let $\hat{b}=A \hat{x}$ and 


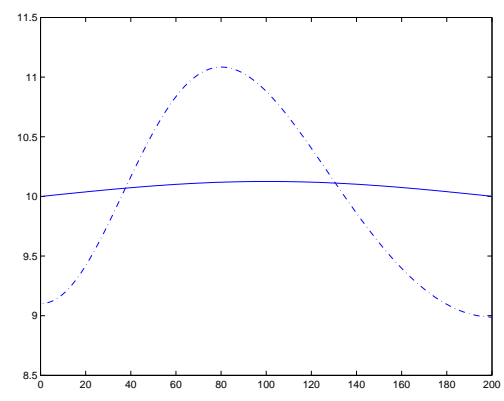

(a)

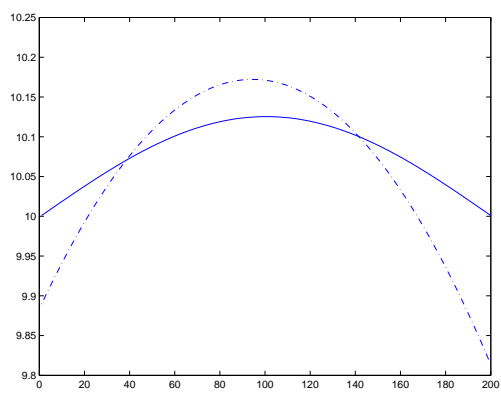

(b)

FIG. 3.3. Example 3.4. Solution $\hat{x}$ to the error-free problem (1.3) (solid curves) and computed approximations (dash-dotted curves); the approximate solutions are $x_{I, \mu}$ in (a) and $x_{L, \mu}$ with $L$ determined by solving Inverse Problem 1 in (b).

determine the error vector $e$ similarly as in Example 3.2. We assume it is known that $b$ has a relative error of $1 \%$. A suitable value of the regularization parameter $\mu$ in (1.5) then can be determined with the aid of the discrepancy principle, i.e., we choose $\mu>0$ so that the solution $x_{L, \mu}$ of the Tikhonov minimization problem (1.5) satisfies

$$
\left\|A x_{L, \mu}-b\right\|=1 \cdot 10^{-2}\|b\| .
$$

Starting with $L=I$ and using the discrepancy principle to determine $\mu>0$ yields the approximate solution $x_{I, \mu}$ of (1.3) with $\left\|x_{I, \mu}-\hat{x}\right\|=6.915 \cdot 10^{-2}\|\hat{x}\|$. Figure 3.3(a) displays $x_{I, \mu}$ and $\hat{x}$. Constructing an improved regularization matrix by solving Inverse Problem 1, using $x_{I, \mu}$ as input, yields the regularization matrix $L$. The approximate solution $x_{L, \mu}$ of (1.1) is computed by solving (1.5) with $\mu>0$ determined by the discrepancy principle. We find $x_{L, \mu}$ to be a significantly better approximation of $\hat{x}$ than $x_{I, \mu}$; we have $\left\|x_{L, \mu}-\hat{x}\right\|=6.357 \cdot 10^{-3}\|\hat{x}\|$. The vector $x_{L, \mu}$ is shown in Figure 3.3(b).

For comparison, we note that the solution $x_{L, \mu}$ for $L$ given by (1.7) and $\mu$ determined by the discrepancy principle has the error $\left\|x_{L, \mu}-\hat{x}\right\|=6.022 \cdot 10^{-1}\|\hat{x}\|$. If we instead use the regularization matrix (1.8), then we obtain $\left\|x_{L, \mu}-\hat{x}\right\|=5.983 \cdot 10^{-1}\|\hat{x}\|$. Thus, the regularization matrix determined by the method of the present paper gives by far the most accurate approximation of $\hat{x}$. However, since $\hat{x}$ is of fairly large norm, so are $b$ and $e$. Consequently, the errors in the computed approximations of $\hat{x}$ are quite large for all the regularization matrices.

4. Conclusion. The choice of regularization matrix is both important and difficult. We propose a novel approach to determine these matrices by solving an inverse matrix problem designed so that the regularization matrix does not damp important features of the desired solution of the discrete ill-posed problem. Computed examples illustrate the good performance of the regularization matrices determined in this manner.

Acknowledgment. The authors would like to thank a referee for comments.

\section{REFERENCES}


[1] M. L. Baart, The use of auto-correlation for pseudo-rank determination in noisy ill-conditioned linear least-squares problems, IMA J. Numer. Anal., 2 (1982), pp. 241-247.

[2] C. Brezinski, M. Redivo-Zaglia, G. Rodriguez, and S. Seatzu, Multi-parameter regularization techniques for ill-conditioned linear systems, Numer. Math., 94 (2003), pp. 203-224.

[3] C. Brezinski, G. Rodriguez, and S. Seatzu, Error estimates for linear systems with application to regularization, Numer. Algorithms, 49 (2008), pp. 85-104.

[4] C. Brezinski, G. Rodriguez, and S. Seatzu, Error estimates for the regularization of least squares problems, Numer. Algorithms, 51 (2009), pp. 61-76.

[5] D. Calvetti, L. Reichel, and A. Shuibi, Invertible smoothing preconditioners for linear discrete ill-posed problems, Appl. Numer. Math., 54 (2005), pp. 135-149.

[6] T. F. Chan, G. H. Golub, and P. Mulet, A nonlinear primal-dual method for total variationbased image restoration, SIAM J. Sci. Comput., 20 (1999), pp. 1964-1977.

[7] H. W. Engl, M. Hanke, and A. Neubauer, Regularization of Inverse Problems, Kluwer, Dordrecht, 1996.

[8] P. C. Hansen, Rank-Deficient and Discrete Ill-Posed Problems, SIAM, Philadelphia, 1998.

[9] P. C. Hansen, Regularization tools version 4.0 for MATLAB 7.3, Numer. Algorithms, 46 (2007), pp. 189-194.

[10] T. Huckle and M. Sedlacek, Data based regularization for discrete deconvolution problems, submitted for publication.

[11] T. Huckle and M. Sedlacek, Tikhonov-Phillips regularization with operator dependent seminorm, preprint.

[12] S. Kindermann, Convergence analysis of minimization-based noise level-free parameter choice rules for linear ill-posed problems, Electron. Trans. Numer. Anal., 38 (2011), pp. 233-257.

[13] S. Morigi, L. Reichel, and F. Sgallari, A truncated projected SVD method for linear discrete ill-posed problems, Numer. Algorithms, 43 (2006), pp. 197-213.

[14] S. Noschese, L. Pasquini, and L. Reichel, The structured distance to normality of an irreducible real tridiagonal matrix, Electron. Trans. Numer. Anal., 28 (2007), pp. 65-77.

[15] S. Noschese, L. Pasquini, and L. Reichel, Tridiagonal Toeplitz matrices: properties and novel applications, Numer. Linear Algebra Appl., in press.

[16] S. Noschese and L. Reichel, The structured distance to normality of banded Toeplitz matrices, BIT, 49 (2009), pp. 629-640.

[17] D. L. Phillips, A technique for the numerical solution of certain integral equations of the first kind, J. ACM, 9 (1962), pp. 84-97.

[18] L. Reichel, F. Sgallari, and Q. Ye, Tikhonov regularization based on generalized Krylov subspace methods, Appl. Numer. Math., in press.

[19] L. Reichel and L. N. Trefethen, Eigenvalues and pseudo-eigenvalues of Toeplitz matrices, Linear Algebra Appl., 162-164 (1992), pp. 153-185.

[20] L. Reichel and Q. Ye, Simple square smoothing regularization operators, Electron. Trans. Numer. Anal., 33 (2009), pp. 63-83.

[21] C. B. Shaw, Jr., Improvements of the resolution of an instrument by numerical solution of an integral equation, J. Math. Anal. Appl., 37 (1972), pp. 83-112.

[22] L. N. Trefethen and M. Embree, Spectra and Pseudospectra, Princeton University Press, Princeton, 2005.

[23] J. Weickert, B. M. H. Romeny, and M. A. Viergever, Efficient and reliable schemes for nonlinear diffusion filtering, IEEE Trans. Image Processing, 7 (1998), pp. 398-410. 